\title{
Epidemiological and clinical characteristics of a familial cluster of COVID-19
}

\section{From the Field}

Cite this article: Sun $\mathrm{Y}$, Tian $\mathrm{L}$, Du X, Wang $\mathrm{H}$, Li Y, Wu R (2020). Epidemiological and clinical characteristics of a familial cluster of COVID19. Epidemiology and Infection 148, e145, 1-6. https://doi.org/10.1017/S0950268820001521

Received: 13 May 2020

Revised: 3 June 2020

Accepted: 11 June 2020

\section{Key words:}

Asymptomatic transmission; coronavirus disease 2019; Outside Wuhan; transmission and clinical characteristics

Author for correspondence:

Yong Sun, E-mail: cysunyong@163.com
Yong Sun ${ }^{1}$ D, Lin Tian², Xiaomei $\mathrm{Du}^{1}$, Hua Wang ${ }^{1}$, Yueshan $\mathrm{Li}^{1}$ and Rangbing $\mathrm{Wu}^{1}$

${ }^{1}$ Department of Endocrinology and Metabolism, Dazhou Central Hopsital, Dazhou, Sichuan, China and

${ }^{2}$ Department of Intensive Care Unit, Dazhou Central Hospital, Dazhou, Sichuan, China

\begin{abstract}
We report a family cluster of severe acute respiratory syndrome coronavirus 2 (SARS-CoV-2) infection involving five patients in a family cluster in Dazhou, China, including the epidemiological, clinical, laboratory and radiological findings. Three-generation transmission was observed. Through epidemiological investigation, we observed asymptomatic transmission to a cohabiting family member, as well as person-to-person transmission of SARS-CoV-2 outside Wuhan city. The asymptomatic transmission demonstrated here provides evidence that there could be a greater risk of Coronavirus Disease 2019 (COVID-19) spread. This cluster also demonstrated that COVID-19 is transmissible during the incubation period of an asymptomatic person. Early isolation and treatment, stressing prevention of cluster outbreaks, could help prevent further spread of the epidemic.
\end{abstract}

(c) The Author(s), 2020. Published by Cambridge University Press. This is an Open Access article, distributed under the terms of the Creative Commons AttributionNonCommercial-ShareAlike licence (http:// creativecommons.org/licenses/by-nc-sa/4.0/), which permits non-commercial re-use, distribution, and reproduction in any medium, provided the same Creative Commons licence is included and the original work is properly cited. The written permission of Cambridge University Press must be obtained for commercial re-use.

\section{CAMBRIDGE} UNIVERSITY PRESS

\section{Introduction}

In December 2019, a series of respiratory illnesses caused by a novel coronavirus, officially named Coronavirus Disease 2019 (COVID-19), was detected in Wuhan city, Hubei Province of China, and this disease spread rapidly around the country and the world. As of 30 March 2020, 693282 patients with COVID-19 have been reported, and 33106 deaths have been confirmed worldwide [1]. The disease is believed to be transmitted mostly via droplets or close contact, and the incubation period ranges from 2 to 14 days [2]. The typical symptoms are fever, dry cough, myalgia and fatigue [2]. COVID-19 has the characteristics of rapid transmission, atypical clinical symptoms and easily affecting both lungs, leading to missed diagnoses and misdiagnoses [3-7].

According to the 'Prevention \& Control Program for Novel Coronavirus Pneumonia (Trial) 6th Edition' enacted by China's National Health Commission [8], close contacts are defined as those who did not use effective protection and had close contact with suspected or confirmed cases 2 days before the onset of symptoms. To date, accumulated evidence has indicated person-to-person transmission to be the cause of most infections [3,9-11]. Li had demonstrated that person-to-person transmission occurred among close contacts since the mid-December 2019 [10].

The massive levels of human movement that occurred during the traditional Chinese New Year holidays fuelled the epidemic. Cases had also been reported in other provinces of China, as well as in other countries with no history of travel to Wuhan, which suggested that local person-to-person transmission was occurring in these areas. At present, a total of 42 confirmed cases of COVID-19 have been reported in Dazhou, Sichuan Province, China, among which are many family cluster cases [12]. With the development of the epidemic, the proportion of clustered outbreaks is increasing. Cluster outbreaks have become a major component of outbreak development throughout the country $[13,14]$. It is important to note that while, at present, the number of new cases has recently been reduced in China, they have increased exponentially in other countries, including South Korea, Italy and the United States [1517], which is a major threat to public health [18]. Here, we report the epidemiological, clinical, radiological and laboratory findings of a family cluster of five patients and provide guidance for the management of this outbreak.

\section{Methods}

\section{Study design and patients}

We performed a retrospective study of a family cluster of severe acute respiratory syndrome coronavirus 2 (SARS-CoV-2) infection involving five patients admitted to the isolation ward of Dazhou Central Hospital in February 2020. This study was reviewed and approved by the Medical Ethics Committee of Dazhou Central Hospital (approval number 2020014). 


\section{Definitions}

Determination of cluster epidemic

In reference to the Guidelines for Epidemiological Survey of Cluster Epidemic of COVID-19 (Tentative, 1st edition), a cluster epidemic refers to 2 or more confirmed cases or asymptomatic infected cases identified in a small area (e.g. a family, a construction site, an organisation) within 14 days, and there is the possibility of person-to-person transmission due to close contact or a possibility of infection due to common exposure.

\section{Epidemiological survey}

In reference to the Guidelines for Epidemiological Survey of Cluster Epidemic of COVID-19 (Tentative, 1st edition), a firstgeneration case normally refers to the earliest affected case, i.e., the first case in the cluster epidemic. A second-generation case should generally meet all of the following criteria: (1) history of contact with the first-generation case within 1-14 days prior to disease onset; (2) no history of travel to or residence in Wuhan or its peripheries or other communities reporting any cases and (3) no history of suspected exposure, such as hospital visit, or no evident community transmission in the area where the case was located. To determine a third- or later-generation case, reference can be made to the criteria for determining a secondgeneration case.

\section{Data collection}

We obtained epidemiological, clinical, laboratory, management and outcome data from the hospital's electronic record system. If data missing from the records were needed, we obtained the data by direct communication with the attending physicians and other health care providers. Throat-swab specimens from the upper respiratory tract were obtained from the patients and sent to the Dazhou Centre for Disease Control and Prevention (CDC) to detect COVID-19 by applying quantitative polymerase chain reaction analysis [3]. All patients were tested for respiratory pathogens and for the nucleic acid of influenza viruses $A$ and B. For each case, computed tomography (CT) was performed upon admission. Laboratory assessments include a complete blood count and serum biochemistry.

\section{Diagnostic standard}

Diagnoses were performed according to the 'Diagnosis \& Treatment Scheme for Novel Coronavirus Pneumonia (Trial) 6th edition' enacted by the National Health Commission of the People's Republic of China [19].

\section{Results}

\section{General information}

The family cluster comprised five patients aged 28-82 years, median age 52 years, including 1 (20\%) patient with underlying diseases (hypertension and coronary heart disease) (Table 1).

\section{Clinical manifestations and epidemiological characteristics}

To better understand the transmission characteristics of COVID-19 outside Wuhan city, we carefully clarified the contact history of each case. Case 1 was a 56-year-old woman who had a history of travel to the city of Wuhan, Hubei province, on 21-22
January 2020 (Table 2 and Fig. 1). On 22 January 2020, case 1 returned to Dazhou and stayed with her mother (case 2), daughter (case 3) and daughter-in-law (case 4) from 23 to 27 January 2020. Case 1 developed fever and cough on 30 January 2020. On 28 January 2020, before case 1 showed any symptoms, case 4 had dinner and stayed with her mother (case 5). Cases 2-5 were all local Dazhou residents who had no history of contact with any patient with confirmed or suspected COVID-19 in the previous 14 days. On 1 February 2020, case 1 visited the hospital and tested positive for SARS-CoV-2 infection. The Dazhou CDC responded immediately and put case 1's family members (cases 2-5) in isolation, and they underwent epidemiological investigations and nucleic acid tests. Case 2, 3 and 5 successively developed fever, cough and fatigue symptoms, and were declared positive for SARS-CoV-2 infection on 4, 2 and 10 February, respectively. The nucleic acid test was also positive for case 4 . however, she had no symptoms of infection, even during hospitalisation. As an asymptomatic patient, case 4 had transmitted the virus to case 5. This cluster demonstrated the existence of a threegeneration transmission chain (Table 2). Of note, one asymptomatic case was found in this study.

\section{Radiological and laboratory findings}

At the time of admission, all the cases had abnormal chest CT findings. As shown in Figure 2, case 1 showed multifocal groundglass opacity (GGO), predominantly involving subpleural regions of both lung CT scans. Case 2 showed patchy GGO of both lungs. Cases 3 and 5 showed multifocal GGO, especially around the outer bands of the lungs on CT scans, which were compatible with changes observed in viral pneumonia. Case 4 had no clinical symptoms, but chest CT showed patchy GGO in the right upper lobe. As shown in Table 1, all the cases had normal or reduced white blood cell counts, and cases $2-5$ developed lymphocytopenia, which was consistent with the main characteristic of viral infection. In terms of inflammation indicators, all the patients on admission presented an elevated serum erythrocyte sedimentation rate (ESR), but no elevated C-reactive protein or procalcitonin. Case 4 had higher levels of bilirubin and lactate dehydrogenase $(\mathrm{LDH})$. The other laboratory test results did not show significant abnormalities.

\section{Discussion}

As of 30 March 2020, more than 80000 laboratory-confirmed cases of infection with SARS-CoV-2 had been reported in China [20]. The early stage of the COVID-19 epidemic was dominated by sporadic cases. With the expansion and spread of the virus, interpersonal and concentrated transmission has occurred in multiple communities and families across the country. With the gradual reduction of imported cases, the number of secondgeneration cases is increasing, especially cases of family clusters. In this study, we provide epidemiological and clinical data on a familial cluster of SARS-CoV-2 infection involving five patients in Dazhou, China.

The symptoms of this novel pneumonia were non-specific. As in previous studies, fever, cough and fatigue were the most common onset symptoms [3-9]. Case 3 in this cluster had diarrhoea but no respiratory symptoms and fever. Case 4 had no symptoms during admission, which increased the difficulty of achieving the diagnosis. We speculated that, during transmission, various transmitters would cause different numbers of secondary cases and 
Table 1. Summary of clinical features and laboratory results of the family cluster infected with COVID-19

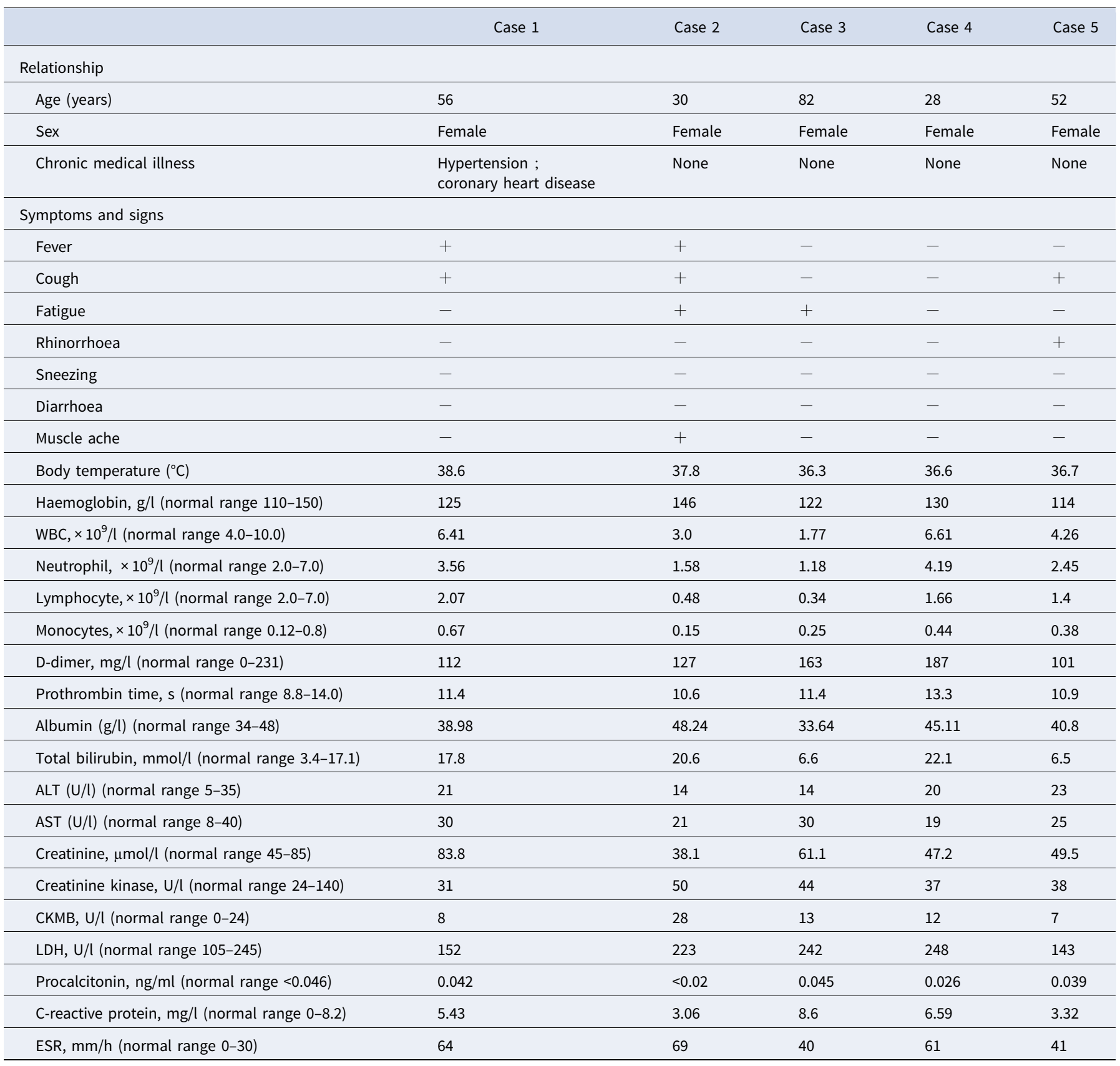

WBC, white blood cell; ALT, alanine aminotransferase; AST, aspartate aminotransferase; CKMB, creatine kinase-MB; LDH, lactate dehydrogenase; ESR, erythrocyte sedimentation rate.

Table 2. Familial cluster outbreak of five COVID-19 cases

\begin{tabular}{lllllll}
\hline Case & Generation & \multicolumn{1}{c}{ Exposure Factor } & Date of Exposure & Date of Onset & Date of Confirmation & $\begin{array}{c}\text { First Contact }- \\
\text { Confirmation Interval (d) }\end{array}$ \\
\hline 1 & First & Sojourn in Wuhan & 21 January 2020 & 30 January 2020 & 1 February 2020 & 11 \\
\hline 2 & Second & Living together with case 1 & 23 January 2020 & 31 January 2020 & 4 February 2020 & 12 \\
\hline 3 & Second & Living together with case 1 & 23 January 2020 & 29 January 2020 & 2 February 2020 & 11 February 2020 \\
\hline 4 & Second & Living together with case 1 & 23 January 2020 & - & 10 February 2020 & 19 \\
\hline 5 & Third & Living together with case 4 & 28 January 2020 & 7 February 2020 & & 13 \\
\hline
\end{tabular}




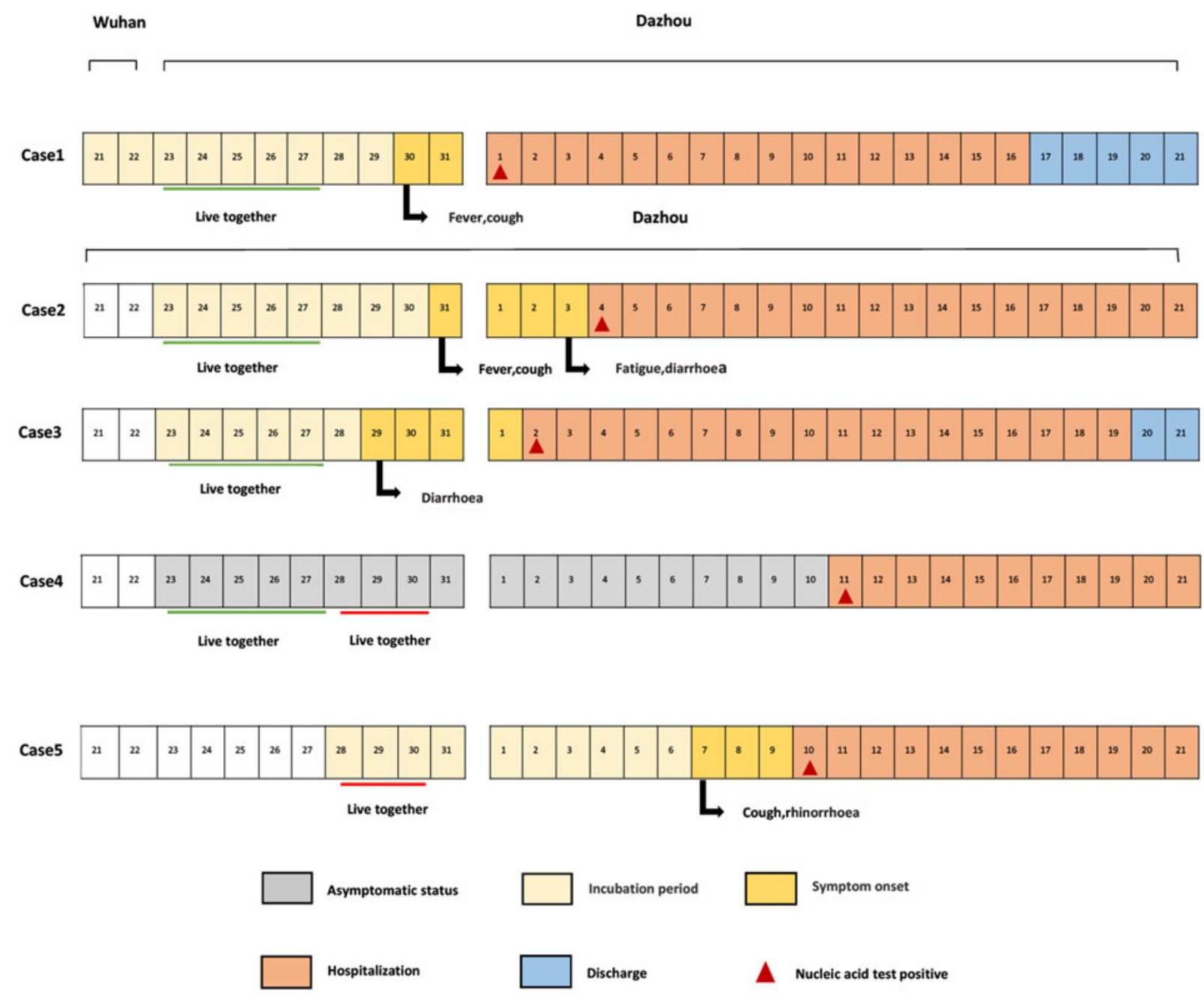

Fig. 1. Relationship between the course and transmission of one case in familial cluster outbreak.

present varied severity levels of the disease. Furthermore, the immune response among patients varies due to great individual differences. Differences in clinical symptoms therefore arise. Nevertheless, these atypical initial symptoms also deserve attention similar to the more common symptoms. For instance, there have been reports of patients with COVID-19 presenting with diarrhoea as the initial symptom of illness onset [21]. Previous studies have found the levels of ESR and LDH increased in asymptomatic patients. In our study, as an asymptomatic patient, laboratory findings of case 4 had abnormalities in indicators such as raised bilirubin, ESR and slightly raised LDH. Notably, compared with symptomatic patients, these indicators soon returned to normal during our follow-up [22, 23]. As with viral pneumonia due to other aetiologies, patients infected by COVID-19 show CT manifestation of GGO in the lungs [3-7, 21, 24].

In this familial cluster outbreak, case 1 had a history of exposure in Wuhan and could be defined as the source of infection for this outbreak. Case 3 had the onset before the primary case; thus, we can conclude that primary cases are infectious in the incubation period. Of the 3063 tourists tested for SARS-CoV-2 on the 'Diamond Princess' 328 had been reported as having asymptomatic cases of coronavirus infection, which suggests a large number of infected individuals with no or slight symptoms [25]. Zou et al. had found that the viral loads of symptomatic and asymptomatic patients were similar, which suggest the transmission potential of asymptomatic patients [26]. We further provide evidence for transmission from an asymptomatic infector to a cohabiting family member that led to COVID-19 pneumonia. These findings indicate that asymptomatic infectors could cause personto-person transmission, and they should therefore be considered sources of COVID-19 infection. By screening close contacts with a nucleic acid test, which is the main diagnostic indicator, we could locate patients, especially asymptomatic carriers as soon as possible.

Currently, with the resumption of business and education, massive crowd, movements and hence contact, inevitably take place. Moreover, the number of imported backflow cases has also been growing, which objectively provides an opportunity for the novel coronavirus to spread. Therefore, although the confirmed cases of COVID-19 have declined, we should continue to maintain the intensity of scrutiny and investigation for potential cases, paying significant attention to the potential risk of clustering among various groups so as to prevent the domestic epidemiological trend from rising for the second time. 

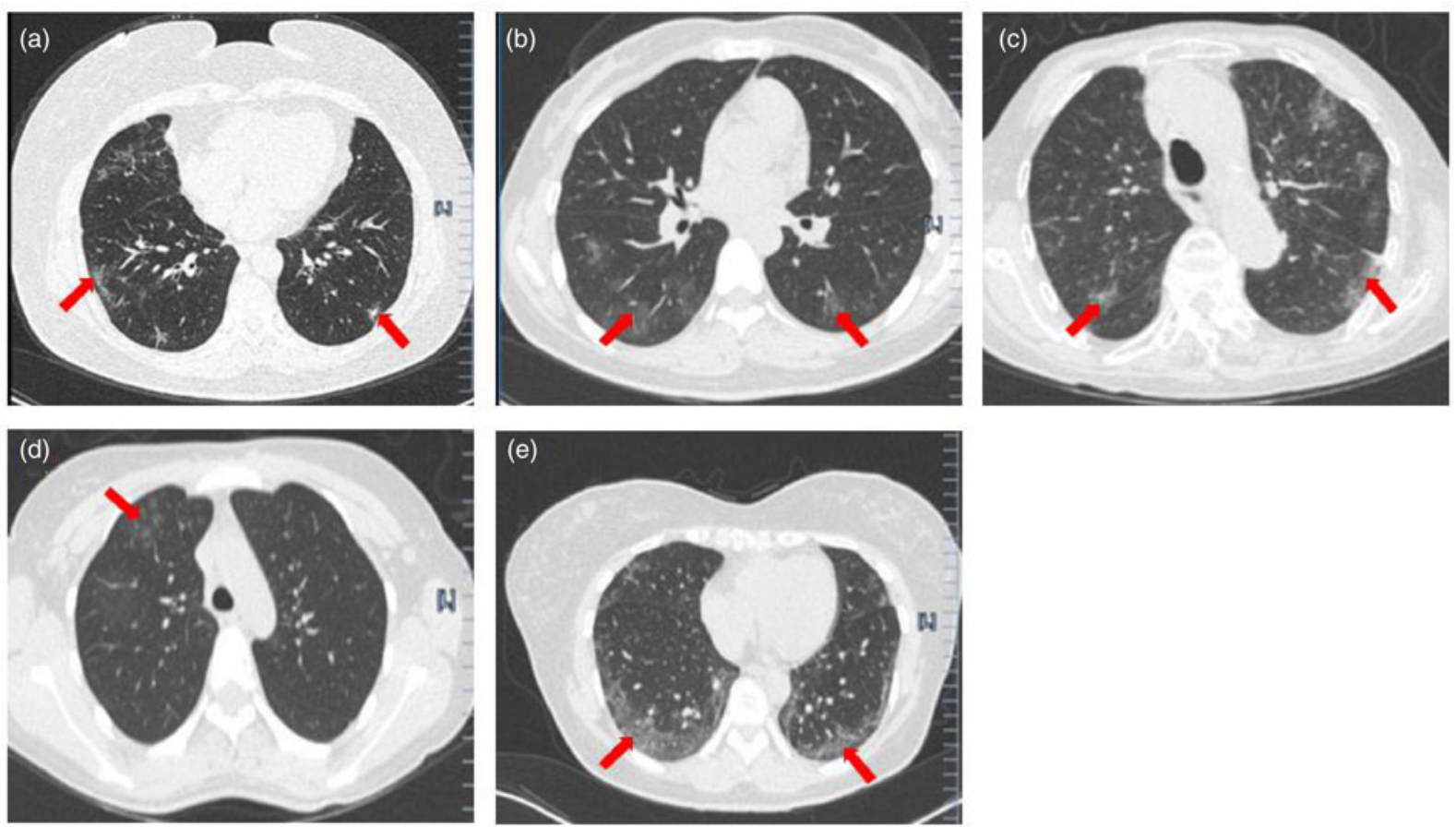

Fig. 2. Chest CT imaging of case 1 (a), case 2 (b), case 3 (c), case 4 (d) and case 5 (E). (a) CT shows multifocal GGO involving the subpleural regions of both lungs (red arrow). (b) CT shows patchy GGOs of both lungs (red arrow). (c and e) CT shows multifocal patchy GGOs that primarily appear at the peripheral area of both lungs (red arrow). (d) CT shows patchy GGOs in the right upper lobe (red arrow).

Acknowledgements. The authors thank Fanwei Zeng, Fanxin Zeng, Chun Liu, Cheng Liu, Wenlai Zhou and Lin Xu (Dazhou Central Hospital) for supporting this study.

\section{Conflict of interest. None.}

Data availability statements. The data that support the findings of this study are available from the corresponding author, S.Y., upon reasonable request.

\section{References}

1. Organization WH (2020) Coronavirus disease 2019 (COVID-19) Situation Report-70. Available at https://www.who.int/docs/default-source/ coronaviruse/situation-reports/20200330-sitrep-70-covid-19.pdf?sfvrsn= 7e0fe3f8_4.

2. Singhal T (2020) A review of coronavirus disease-2019 (COVID-19). Indian Journal of Pediatrics 87, 281-286.

3. Huang C et al. (2020) Clinical features of patients infected with 2019 novel coronavirus in Wuhan, China. Lancet 395, 497-506.

4. Xu XW et al. (2020) Clinical findings in a group of patients infected with the 2019 novel coronavirus (SARS-Cov-2) outside of Wuhan, China: retrospective case series. British Medical Journal 368, m606.

5. Chen $\mathbf{N}$ et al. (2020) Epidemiological and clinical characteristics of 99 cases of 2019 novel coronavirus pneumonia in Wuhan, China: a descriptive study. Lancet 395, 507-513.

6. Wang D et al. (2020) Clinical characteristics of 138 hospitalized patients with 2019 novel coronavirus-infected pneumonia in Wuhan, China. The Journal of American Medical Association 323, 1061-1069.

7. Chang D et al. (2020) Epidemiologic and clinical characteristics of novel coronavirus infections involving 13 patients outside Wuhan, China. The Journal of American Medical Association 323, 1092-1093.

8. National Health Commission of the People's Republic of China (2020) New Coronavirus Pneumonia Prevention and Control Program (version 6). Beijing, China: National Health Commission of the People's Republic of China. Available at http://www.nhc.gov.cn/jkj/s3577/202003/4856d5b0458 141fa9f376853224d41d7/files/4132bf035bc242478a6eaf157eb0d979.pdf.
9. Chan JF et al. (2020) A familial cluster of pneumonia associated with the 2019 novel coronavirus indicating person-to-person transmission: a study of a family cluster. Lancet 395, 514-523.

10. Li Q et al. (2020) Early transmission dynamics in Wuhan, China, of novel coronavirus-infected pneumonia. The New England Journal of Medicine 382, 1199-1207.

11. Phan L T et al. (2020) Importation and human-to-human transmission of a novel coronavirus in Vietnam. The New England Journal of Medicine 382, 872-874.

12. Sichuan Center For Disease Control And Prevention. Available at https://www.sccdc.cn/View.aspx?id=19786.

13. Special Expert Group for Control of the Epidemic of Novel Coronavirus Pneumonia of the Chinese Preventive Medicine Association (2020) An update on the epidemiological characteristics of novel coronavirus pneumonia (COVID-19). Zhonghua Liu Xing Bing Xue Za Zhi 41, 139-144.

14. Novel Coronavirus Pneumonia Emergency Response Epidemiology Team (2020) The epidemiological characteristics of an outbreak of 2019 novel coronavirus diseases (COVID-19) in China. Zhonghua Liu Xing Bing Xue Za Zhi 41, 145-151.

15. Ki M and Task Force for 2019-nCoV (2020) Epidemiologic characteristics of early cases with 2019 novel coronavirus (2019-nCoV) disease in Republic of Korea. Epidemiology and Health 42, e2020007.

16. Giovanetti $\mathbf{M}$ et al. (2020) The first two cases of 2019-nCoV in Italy: where they come from? Journal of Medical Virology 92, 518-521.

17. CDC COVID-19 Response Team (2020) Severe outcomes among patients with coronavirus disease 2019 (COVID-19) - United States, 12 February-16 March 2020. Morbidity and Mortality Weekly Report 69, 343-346.

18. Deng SQ and Peng HJ (2020) Characteristics of and public health responses to the coronavirus disease 2019 outbreak in China. Journal of Clinical Medicine 9, 575.

19. National Health Commission of the People's Republic of China and National Administration of Traditional Chinese Medicine. Diagnosis \& Treatment Scheme for Novel Coronavirus Pneumonia (Trial), 6th Edn. National Health Commission of the People's Republic of China and National Administration of Traditional Chinese Medicine, available at: http://www.nhc.gov.cn/yzygj/s7653p/202002/8334a8326dd94d329df351 d7da8aefc2.shtml. 
20. Chinese Center for Disease Control and Prevention. Available at http:// www.chinacdc.cn/jkzt/crb/zl/szkb_11803/jszl_11809/202003/t20200331_ 215669.html.

21. Wang $\mathbf{Z}$ et al. (2020) Clinical features of 69 cases with coronavirus disease 2019 in Wuhan, China. Clinical Infectious Diseases pii, ciaa272.

22. Ma Y et al. (2020) Characteristics of asymptomatic patients with SARS-CoV-2 2 infection in Jinan, China. Microbes and Infection 22, 212-217.

23. Wu J et al. (2020) Clinical features and outcomes of asymptomatic cases of SARS-CoV-2 infection. The Journal of Infection 53, 30238-3.
24. Xu X et al. (2020) Imaging and clinical features of patients with 2019 novel coronavirus SARS-CoV-2. European Journal of Nuclear Medicine and Molecular Imaging 47, 1275-1280.

25. Mizumoto K et al. (2020) Estimating the asymptomatic proportion of coronavirus disease 2019 (COVID-19) cases on board the Diamond Princess cruise ship, Yokohama, Japan, 2020. Eurosurveillance 25, 1560 7917.

26. Zou L et al. (2020) SARS-CoV-2 viral load in upper respiratory specimens of infected patients. The New England Journal of Medicine 382, 11771179 . 